IOSR Journal of Engineering

e-ISSN: 2250-3021, p-ISSN: 2278-8719,

Vol. 2, Issue 12 (Dec. 2012), ||V3\| PP 41-44

\title{
On contact slant submanifolds in Kenmotsu space forms
}

\author{
Pradeep Kumar Pandey \\ Department of Mathematics, Jaypee University of Information Technology, Waknaghat, Solan, Himachal- \\ 173234
}

\begin{abstract}
In this paper a sharp estimate of the maximum Ricci curvature function for a special contact slant submanifold $M$ in a Kenmotsu space form $\bar{M}(c)$, in terms of the main extrinsic invariant namely the squared mean curvature has been obtained. If submanifold $M$ satisfies the equality case identically, then it is minimal.
\end{abstract}

Mathematics Subject Classification 2000: 53C40, 53B25, 53D15.

Keywords: Contact slant submanifold, Kenmotsu space form, Mean curvature, Ricci curvature.

\section{INTRODUCTION}

The study of slant submanifold of an almost Hermitian manifold was started by B. Y. Chen ([1], [2]). Further Chen and Tazawa ([3], [4]), furnished examples of slant submanifolds of $C^{2}$ and $C^{4}$, while Maeda, Ohnita and Udagawa [5], studied slant submanifolds of Kaehler manifold. The notion of slant submanifold of almost contact metric manifold was introduced by A. Lotta [6]. A class of manifolds was studied and characterized by K. Kenmotsu [7], and the structure so obtained is nowadays known as Kenmotsu structure. In general a Kenmotsu structure is not Sasakian. A Kenmotsu space form is a Kenmotsu manifold with constant $\phi$-holomorphic sectional curvature $c$, denoted by $\bar{M}(c)$. Recently, K. Arslan et. al . [8], have established a sharp relationship between the Ricci curvature and the squared mean curvature for submanifolds in Kenmotsu space forms. A $(2 \mathrm{~m}+1)$-dimensional Riemannian manifold $\bar{M}$ is said to be an almost contact metric manifold if there exist structure tensors $(\phi . \xi, \eta, g)$, where $\phi$ is a $(1,1)$ tensor field, $\xi$ a vector field, $\eta$ a 1 form and g the Riemannian metric on $\bar{M}$ satisfying [9]:

$$
\phi^{2} X=-X+\eta(X) \xi
$$

$(1.3)$

(1.4) $\quad \eta(\phi X)=0$

(1.5) $\quad g(\phi X, \phi Y)=g(X, Y)-\eta(X) \eta(Y)$

(1.6) $\quad \eta(X)=g(X, \xi)$

for any $X, Y \in \overline{T M}$, where $\overline{T M}$ denotes the Lie algebra of vector fields on $\bar{M}$. An almost contact metric manifold $\bar{M}$ is called a Kenmotsu manifold if [7],

$$
\left(\bar{\nabla}_{X} \phi\right) Y=-g(X, \phi Y) \xi-\eta(Y) \phi X \quad \text { and } \quad \bar{\nabla}_{X} \xi=X-\eta(X) \xi
$$


Where $\bar{\nabla}$ denotes the Levi-Civita connection on $\bar{M}$.

The curvature tensor $\bar{R}$ of a Kenmotsu space form $\bar{M}(c)$ is given by [7],

$$
\begin{aligned}
\bar{R}(X, Y) Z=\frac{c-3}{4}\{g(Y, Z) X-g(X, Z) Y\}+\frac{c+1}{4}\{\eta(X) \eta(Z) Y \\
\quad-\eta(Y) \eta(Z) X+\eta(Y) g(X, Z) \xi-\eta(X) g(Y, Z) \xi \\
\quad-g(\phi X, Z) \phi Y+g(\phi Y, Z) \phi X+2 g(X, \phi Y) \phi Z\}
\end{aligned}
$$

for all $X, Y, Z \in \overline{T M}$.

Let $\mathrm{M}$ be an n-dimensional submanifold of a Kenmotsu space form $\bar{M}(c)$ of constant $\phi$-sectional curvature $c$. We denote by $K(\pi)$ the sectional curvature of $M$ associated with a plane section $\pi \subset T_{p} M$, $p \in M$, and $\nabla$ the Riemannian connection on $M$, respectively. We denote by $h$ the second fundamental form of

$M$ and by $R$ and $\bar{R}$ the curvature tensors of the submanifold $M$ and Kenmotsu space form $\bar{M}(c)$, respectively. Then the equation of Gauss is given by

$$
\bar{R}(X, Y, Z, W)=R(X, Y, Z, W)+g(h(X, W), h(Y, Z))-g(h(X, Z), h(Y, W))
$$

for all $X, Y, Z, W \in T M$.

Let $p \in M$ and $\left\{e_{1}, e_{2}, \ldots, e_{2 m+1}\right\}$ be an orthonormal basis of the tangent space $\overline{T_{p} M}$, such that $e_{1}, e_{2}, \ldots, e_{n}$ are tangent to $M$ at $p$. Let $H$ denote the mean curvature vector, that is $H(p)=\frac{1}{n} \sum_{i=1}^{n} h\left(e_{i}, e_{i}\right)$. Now we set

$$
h_{i, j}^{r}=g\left(h\left(e_{i}, e_{j}\right), e_{r}\right), \quad i, j=\{1,2, \ldots, n\}, \quad r \in\{(n+1), . .,(2 m+1)\}
$$

For any vector field $X$ tangent to $M$, we put $\phi X=P X+F X$, where $P X$ and $F X$ denote the tangential and normal components of $\phi X$ respectively.

\section{CONTACT SLANT SUBMANIFOLDS}

K. Arslan et. al. [8] have established a sharp relationship between the Ricci curvature and the squared mean curvature for submanifolds in Kenmotsu space forms. We recall the following theorem for our ready reference.

Theorem [8]: Let $\bar{M}(c)$ be a $(2 \mathrm{~m}+1)$-dimensional Kenmotsu space form and $M$ an n-dimensional submanifold tangent to $\xi$. Then

(i) for each unit vector $X \in T_{p} M$ orthogonal to $\xi$,

$$
\operatorname{Ric}(X) \leq \frac{1}{4}\left\{(n-1)(c-3)+\frac{1}{2}\left(3\|P X\|^{2}-2\right)(c+1)+n^{2}\|H\|^{2}\right\}
$$


(ii) A unit tangent vector $X$ orthogonal to $\xi$ at $p$ satisfies the equality case of (2.1) if and only if

$$
\left\{\begin{array}{l}
h(X, Y)=0, \forall Y \in T_{p} M \text { orthogonal to } X \\
2 h(X, X)=n H(p)
\end{array}\right.
$$

A submanifold $M$ tangent to $\xi$ in a Kenmotsu space form $\bar{M}(c)$ is said to be contact slant submanifold if for any $p \in M$ and any $X \in T_{p} M$ linearly independent on $\xi$, the angle between $\phi X$ and $T_{p} M$ is a constant $\theta$, called the slant angle of $M$. A contact slant submanifold which is neither invariant nor anti-invariant is called a proper slant submanifold.

A particular case of contact slant submanifolds, which we call special contact slant submanifolds ([10], [13]). A proper contact $\theta$-slant submanifold is said to be special contact slant if

$$
\left(\nabla_{X} P\right) Y=g(Y, P X) \xi-\eta(Y) P X
$$

Any 3-dimensional proper contact slant submanifold of a Kenmotsu manifold is a special contact slant submanifold [10].

We denote by $\mathbf{R}$ the maximum Ricci curvature function on $M$ [11], defined by

$$
\mathbf{R}(p)=\max \left\{\operatorname{Ric}(u) \mid u \in T_{p}^{1} M\right\}, p \in M
$$

where $T_{p}^{1} M=\left\{u \in T_{p} M \mid g(u, u)=1\right\}$.

If $n=3, \mathbf{R}$ is the Chen's first invariant $\delta_{M}$ introduced in [12]. For $n>3, \mathbf{R}$ is the Chen's invariant $\delta(n-1)$.

In this section, we establish an inequality for the Chen invariant $\mathbf{R}$ and show that any special contact slant submanifold which satisfies the equality case identically is minimal.

Now we show

Theorem (2.1): Let $M$ be an (n+1)-dimensional special contact slant submanifold in a (2n+1)-dimensional Kenmotsu space form $\bar{M}(c)$ of constant $\phi$-sectional curvature $c$. Then for each unit vector $X$ orthogonal to $\xi$, we have

$$
\mathbf{R} \leq \frac{1}{4}\left\{(n+1)^{2}\|\mathrm{H}\|^{2}+\mathrm{n}(\mathrm{c}-3)+\frac{1}{2}\left(3 \cos ^{2} \theta-2\right)(\mathrm{c}+1)\right\}
$$

If $M$ satisfies the equality case in (2.3) identically, then $M$ is a minimal submanifold.

Proof: The inequality (2.3) is an immediate consequence of the inequality (2.1). To ascertain minimality, we assume that $M$ is a special contact slant submanifold of Kenmotsu space form $\bar{M}(c)$, which satisfies the equality case of (2.3) at a point $p \in M$. Now without loss of generality we may choose an orthonormal basis $\left\{e_{0}=\xi, e_{1}, \ldots, e_{n}\right\}$ of $T_{p} M$ such that $\mathbf{R}(p)=\operatorname{Ric}\left(e_{n}\right)$. Then by (2.2) it follows that

$$
h\left(e_{i}, e_{n}\right)=0, \forall i \in\{0, \ldots, n-1\} ; h\left(e_{n}, e_{n}\right)=\sum_{i=1}^{n-1} \mathrm{~h}\left(\mathrm{e}_{\mathrm{i}}, \mathrm{e}_{\mathrm{i}}\right)
$$

where $\left\{e_{0}=\xi, e_{1}, \ldots, e_{n}, e_{n+1}, \ldots, e_{2 n}\right\}$ is the orthonormal basis with $e_{n+j}=(\sec \theta) P e_{j}$, $j \in\{1,2, \ldots, n\}$ and $e_{n+1}$ parallel to the mean curvature vector $H(p)$. 
Let $A$ denote the Weingarten map of $M$ in $\bar{M}(c)$. Then it is easy to show that $A_{\phi X} Y=A_{\phi Y} X$ for all vector fields $X, Y$ tangent to $M$ (see [10]). Hence we have $h_{i, j}^{n+k}=h_{i, j}^{n+j}=h_{i, j}^{n+i}$ for any $i, j, k \in\{1,2, \ldots, n\}$. Now using equations (2.4) a simple computation yields $H(p)=0$, therefore $M$ is a minimal submanifold. We have the following characterization of $\mathrm{n}$-dimensional special slant submanifolds in the sphere $S^{2 n+1}$ which satisfy the equality case of (2.3) identically.

Theorem (2.2): Let $M$ be an (n+1)-dimensional special contact slant submanifold of the (2n+1)-dimensional sphere $S^{2 n+1}$. Then $M$ satisfies the equality case of (2.3) identically if and only if $M$ is minimal and ruled. Proof: The theorem (2.1) implies that $M$ is a minimal submanifold. From the theorem [8], it is obvious that the equality case of (2.3) holds identically if and only if the relative null space

$$
\mathbf{N}_{p}=\left\{X \in T_{p} M \mid h(X, Y)=0, \forall Y \in T_{p} M\right\} \neq\{0\}
$$

at any $p \in M$.

Let $U_{l}$ denotes interior of the subset consisting of points in $M$ such that the relative null space at $p$ has dimension $l$. It follows that for some $l, U_{l} \neq \varphi$, where $l \in\{1, \ldots, n\}$.

Let $X, Y \in N$, and $Z$ any vector field tangent to $M$. By applying Codazzi equation

$$
\left(\bar{\nabla}_{X} h\right)(Y, Z)=\left(\bar{\nabla}_{Z} h\right)(X, Y),
$$

we get $h\left(\nabla_{X} Y, Z\right)=0$. Then $N$ is integrable on $U_{l}$ and each leaf of $N \mid U_{l}$ is an $l$-dimensional totally geodesic submanifold of $S^{2 n+1}$. Thus submanifold $M$ contains a geodesic of $S^{2 n+1}$ through each point $p \in U_{l}$.

Since $M$ is the union of the closure of all $U_{l}$, we conclude by continuity that $M$ contains a geodesic of the ambient space through each point in $M$. Hence, $M$ is minimal and ruled.

The converse follows from straightforward computation.

\section{REFERENCES}

1). B. Y. Chen, Slant immersions, Bulletin of the Australian Mathematical Society, 41, 1990, 135-147.

2). B. Y. Chen, Geometry of slant submanifolds (Katholieke Universiteit. Leuven, 1990).

3). B. Y. Chen, and Y. Tazawa, Slant submanifolds in complex Euclidean spaces, Tokyo Journal of Mathematics, 14 (1), 1991, 101-120.

4). B. Y. Chen, and Y. Tazawa, Slant surfaces with codimension 2, Ann. Fac. Sci. Toulouse Math. XI (3), 1990, 29-43.

5). S. Maeda, Y. Ohnita, and S. Udagawa, On slant immersions into Kaehler manifolds, Kodai Mathematical Journal, 16, 1993, 205-219.

6). A. Lotta, Slant submanifolds in contact geometry, Bull. Math. Soc. Roumanie, 39, 1996, 183-198.

7). K. Kenmotsu, A class of almost contact Riemannian manifolds, Tohoku Mathematical Journal, 21, 1972, 93-103.

8). K. Arslan, R. Ezentas, I. Mihai, C. Murathan and C. Özgür, Ricci curvature of submanifolds in Kenmotsu space forms, IJMMS 29 (12), 2002, 719-726.

9). D. E. Blair, Contact manifolds in Riemannian geometry (Lecture notes in Mathematics, Springer Verlag 509, 1976, Berlin-New York).

10). R. S. Gupta, S. M. Khursheed and M. H. Shahid, Slant submanifolds of a Kenmotsu manifold, Radovi Matematicki 12, 2004, 205-214.

11). B. Y. Chen, On Ricci curvature of isotropic and Lagrangian submanifolds in complex space forms, Archiv. Math., 74, 2000, 154-160.

12). B. Y. Chen, some pinching and classification theorems for minimal submanifolds, Arch. Math., 60, 1993, 568-578.

13). I. Mihai, and V. Ghisoiu, Minimality of certain contact slant submanifolds in Sasakian space forms, Int. J. Pure and Appl. Math. Sci., 1, 2004, 95-99. 